\title{
Semantics of Fuzzy Sets in Rough Set Theory
}

\author{
Y.Y. Yao \\ Department of Computer Science \\ University of Regina \\ Regina, Saskatchewan \\ Canada S4S 0A2 \\ E-mail: yyao@cs.uregina.ca \\ URL: http://www.cs.uregina.ca/ yyao
}

\begin{abstract}
The objective of this chapter is to provide a semantic framework for fuzzy sets in the theory of rough sets. Rough membership functions are viewed as a special type of fuzzy membership functions interpretable using conditional probabilities. The relationships between fuzzy membership functions and rough membership functions, between core and support of fuzzy set theory and lower and upper approximation of rough set theory, are investigated. It is demonstrated that both theories share the same qualitative properties. Interpretations of fuzzy sets in rough set theory lead to constraints on membership values. Two types of constraints on membership values are studies, namely, constraints on membership values of related elements and constraints on membership values of related sets. The classical rough set model and generalized rough set models are discussed.
\end{abstract}

\section{Introduction}

The theory of rough sets deals with the approximation of sets under indiscernibility $[7,8]$. The theory of fuzzy sets handles vague concepts by allowing partial memberships [23]. Both theories are motivated by the practical needs to manage and process uncertainty inherent in real world problem solving. They are different and complementary generalizations of classical set theory $[2,10,14]$.

Semantic interpretation of a fuzzy membership grade is one of the fundamental issues of fuzzy set theory [4]. Dubois and Prade examined three main semantics of membership functions, namely, similarity, preference and uncertainty [3, 4]. Each of them underlies a particular class of applications. The co-existence of multiple interpretations shows the richness of the concept of fuzzy sets.

The similarity based semantics relies on relationships between objects of a universe in terms of a similarity, a dissimilarity, or a distance function. The membership of an object is the degree of proximity of the object to the prototype elements of a fuzzy set [4]. The similarity based semantics imposes certain qualitative and quantitative constraints, reflecting the similarity of objects. The more similar an object is to the prototype elements of a fuzzy set, the greater is its membership grade, and vice versa. The membership grades can be computed 
by means of an increasing function of the similarity between an object and the prototype elements of a fuzzy set $[3,4]$.

The theory of rough sets is based on a well studied and practical notion called equivalence relations. Equivalence relations are defined and explained in terms of an information table, such as a database. By focusing on one particular type of uncertainty, namely, the discernibility or indistinguishability of elements, rough set theory has a systematic treatment of its semantic interpretations. The formulation and interpretation of rough sets as defined by rough membership functions, set-theoretic operators, as well as rough set approximation operators are inseparable parts of the theory.

Equivalence relations describe a specific type of similarity between objects. Other types of binary relations are also used in generalizations of rough sets [20]. A question arises naturally is whether we can adopt the methodologies used in the study of rough sets to investigate fuzzy sets. We attempt to provide a positive answer to the question in this chapter. Similarity based semantics of fuzzy sets is discussed in terms of rough sets. While many investigations of fuzzy sets focus on quantitative study of the theory, we focus on the qualitative aspects of the theory. Rough membership functions are viewed as a special type of fuzzy membership functions interpretable using conditional probabilities. Rough set approximations are related to the core and support of a fuzzy set. Based on these connections, we demonstrate that both theories share the same qualitative features. We also study qualitative constraints on membership values and approximation operators based on discernibility and similarity of elements. The constraints and approximation operators are studied in detail with respect to the classical rough set model based on equivalence relations or partitions, generalized rough set models based on arbitrary binary relations, and generalized rough set models based on coverings [15].

\section{A Critical Review of Fuzzy Sets}

The notion of fuzzy sets provides a convenient tool for representing vague concepts by allowing partial memberships [23]. Depending on the characteristics of membership values, two types of fuzzy sets have been studied [6]. If fuzzy membership functions take a single value from the unit interval $[0,1]$ or a lattice, the corresponding fuzzy sets are known as type 1 fuzzy sets. If fuzzy membership functions take type 1 fuzzy sets as their values, the corresponding fuzzy sets are known as type 2 fuzzy sets.

In this chapter, we restrict our discussion to the classical fuzzy sets, i.e., the type 1 fuzzy sets. Furthermore, we assume that the universe, namely, the referential set on which fuzzy sets are defined, is finite. For simplicity, we use the same symbols for both standard set-theoretic relations and operators and fuzzy set-theoretic relations and operators. 


\subsection{Fuzzy set systems}

Let $U$ be a finite and non-empty set called universe. A fuzzy subset of $U$ is defined by a membership function:

$$
\mu_{\mathcal{A}}: U \longrightarrow[0,1] .
$$

Fuzzy set inclusion and equality can be defined component-wise. A fuzzy set $\mu_{\mathcal{A}}$ is a subset of another fuzzy set $\mu_{\mathcal{B}}$, written $\mu_{\mathcal{A}} \subseteq \mu_{\mathcal{B}}$ if and only if $\mu_{\mathcal{A}}(x) \leq \mu_{\mathcal{B}}(x)$ for all $x \in U$. Fuzzy set $\mu_{\mathcal{A}}$ is equal to fuzzy set $\mu_{\mathcal{B}}$, written $\mu_{\mathcal{A}}=\mu_{\mathcal{B}}$ if and only if $\mu_{\mathcal{A}}(x)=\mu_{\mathcal{B}}(x)$ for all $x \in U$. Obviously, $\mu_{\mathcal{A}}=\mu_{\mathcal{B}}$ if and only if $\mu_{\mathcal{A}} \subseteq \mu_{\mathcal{B}}$ and $\mu_{\mathcal{B}} \subseteq \mu_{\mathcal{A}}$.

There are many definitions for fuzzy set complement, intersection, and union. The standard min-max system proposed by Zadeh is given component-wise by [23]:

$$
\begin{aligned}
\mu_{\neg \mathcal{A}}(x) & =1-\mu_{\mathcal{A}}(x), \\
\mu_{\mathcal{A} \cap \mathcal{B}}(x) & =\min \left(\mu_{\mathcal{A}}(x), \mu_{\mathcal{B}}(x)\right), \\
\mu_{\mathcal{A} \cup \mathcal{B}}(x) & =\max \left(\mu_{\mathcal{A}}(x), \mu_{\mathcal{B}}(x)\right) .
\end{aligned}
$$

A crisp subset of $U$ may be viewed as a degenerated fuzzy set. In this case, the membership function is the characteristic function taking only two values 0 and 1. The min-max fuzzy set-theoretic operators reduce to the classical set-theoretic operators when characteristic functions are used.

In general, one may define fuzzy set operators using triangular norms (tnorms) and conorms (t-conorms) $[1,5,6]$. A t-norm is a function from $[0,1] \times[0,1]$ to $[0,1]$ and satisfies the following conditions: for $a, b, c \in[0,1]$,

$$
\begin{aligned}
\text { (i). } & \text { Boundary conditions } \\
& t(0,0)=0 \\
& t(1, a)=t(a, 1)=a ; \\
\text { (ii). } \quad & \text { Monotonicity } \\
& (a \leq c, b \leq d) \Longrightarrow t(a, b) \leq t(c, d) ; \\
\text { (iii). } \quad & \text { Symmetry } \\
& t(a, b)=t(b, a) ; \\
\text { (iv). } \quad \text { Associativity } & t(a, t(b, c))=t(t(a, b), c)) .
\end{aligned}
$$

For clarity, some of the conditions are explicitly listed, although they can be obtained from other conditions. For example, the symmetry implies $t(1, a)=$ $t(a, 1)$. We only need either $t(a, 1)=a$ or $t(1, a)=a$. The condition $t(0,0)=0$ follows from $t(1, a)=a$, the symmetry and the monotonicity. The boundary conditions ensure that a t-norm reduces to standard set intersection when only crisp sets are involved. The monotonicity suggests that fuzzy set intersection keep the monotonicity of set intersection with respect to set inclusion. Another version of monotonicity is given by $b \leq c \Longrightarrow t(a, b) \leq t(a, c)$. 
Some commonly used t-norms are $t_{\mathrm{b}}(a, b)=\max (0, a+b-1), t_{\min }(a, b)=$ $\min (a, b)$, the product operator $t_{\mathrm{p}}(a, b)=a b$, and $t_{\mathrm{w}}$ defined by boundary conditions and $t_{\mathrm{w}}(a, b)=0, \forall(a, b) \in[0,1) \times[0,1)$. These t-norms are related by inequality $[1,6]$ :

$$
t_{\mathrm{w}}(a, b) \leq t_{\mathrm{b}}(a, b) \leq t_{\mathrm{p}}(a, b) \leq t_{\min }(a, b) .
$$

Moreover, any t-norm is bounded by $t_{\mathrm{w}}$ and $t_{\mathrm{min}}$, i.e.,

$$
t_{\mathrm{w}}(a, b) \leq t(a, b) \leq t_{\min }(a, b) .
$$

It provides the bounds of the membership values of the intersection of two fuzzy sets.

Suppose $n:[0,1] \longrightarrow[0,1]$ is an operator called negation. With respect to a negation operator, the dual of a t-norm is given by $n(t(n(a), n(b)))$ and called a t-conorm, which is a function $s$ mapping $[0,1] \times[0,1]$ to $[0,1]$ and satisfying the boundary conditions:

$$
\begin{aligned}
\left(\mathrm{i}^{\prime}\right) . & \text { Boundary conditions } \\
& s(1,1)=1, \\
& s(a, 0)=s(0, a)=a,
\end{aligned}
$$

and conditions of monotonicity, symmetry, and associativity. Suppose the negation operator is defined by $n(a)=1-a$. The t-conorm $s$ corresponding to a t-norm $t$ is given by:

$$
\begin{aligned}
s(a, b) & =n(t(n(a), n(b))) \\
& =1-t(1-a, 1-b) .
\end{aligned}
$$

The t-conorms of $t_{\min }, t_{\mathrm{p}}$ and $t_{\mathrm{b}}$ are $s_{\max }(a, b)=\max (a, b), s_{\mathrm{p}}(a, b)=a+b-a b$, and $s_{\mathrm{b}}(a, b)=\min (1, a+b)$, respectively. The t-conorm corresponding to $t_{\mathrm{w}}$ is given by the boundary conditions and $s_{\mathrm{w}}(a, b)=1, \forall(a, b) \in(0,1] \times(0,1]$. Similarly, we have:

$$
s_{\max }(a, b) \leq s_{\mathrm{p}}(a, b) \leq s_{\mathrm{b}}(a, b) \leq s_{\mathrm{w}}(a, b) .
$$

Any t-conorm is bounded by $s_{\max }$ and $s_{\mathrm{w}}$ :

$$
s_{\max }(a, b) \leq s(a, b) \leq s_{\mathrm{w}}(a, b) .
$$

It provides the bounds of the membership values of the union of two fuzzy sets. Combining with equation (4), we have:

$$
t(a, b) \leq \min (a, b) \leq \max (a, b) \leq s(a, b),
$$

which expresses the connection between fuzzy set intersection and union.

Let $t$ and $s$ be a pair of t-norm and t-conorm. We define fuzzy set intersection and union component-wise by:

$$
\begin{aligned}
& \mu_{\mathcal{A} \cap \mathcal{B}}(x)=t\left(\mu_{\mathcal{A}}(x), \mu_{\mathcal{B}}(x)\right), \\
& \mu_{\mathcal{A} \cup \mathcal{B}}(x)=s\left(\mu_{\mathcal{A}}(x), \mu_{\mathcal{B}}(x)\right) .
\end{aligned}
$$


An important feature of fuzzy set operators as defined by t-norms and t-conorms is that they are truth-functional operators. In other words, membership functions of complement, intersection, and union of fuzzy sets are defined based solely on the membership functions of the fuzzy sets involved [14].

\subsection{Qualitative characterization of fuzzy sets}

Although the conditions on t-norms and t-conorms are expressed in quantitative forms, they are of qualitative nature that characterize a large class of functions. From t-norms, one can easily obtain the following qualitative conditions:

$$
\begin{aligned}
& \text { (I). Boundary conditions } \\
& \mu_{\emptyset \cap \emptyset}=\emptyset \text {, } \\
& \mu_{U \cap \mathcal{A}}=\mu_{\mathcal{A} \cap U}=\mu_{\mathcal{A}}, \\
& \text { (II). Monotonicity } \\
& {\left[\mu_{\mathcal{A}} \subseteq \mu_{\mathcal{C}}, \mu_{\mathcal{B}} \subseteq \mu_{\mathcal{D}}\right] \Longrightarrow \mu_{\mathcal{A} \cap \mathcal{B}} \subseteq \mu_{\mathcal{C} \cap \mathcal{D}}} \\
& \text { (III). Commutativity } \\
& \mu_{\mathcal{A} \cap \mathcal{B}}=\mu_{\mathcal{B} \cap \mathcal{A}}, \\
& \mu_{(\mathcal{A} \cap \mathcal{B}) \cap \mathcal{C}}=\mu_{\mathcal{A} \cap(\mathcal{B} \cap \mathcal{C})} .
\end{aligned}
$$

For the t-conorms, we have:

$$
\begin{aligned}
\left(\mathrm{I}^{\prime}\right) . & \text { Boundary conditions } \\
& \mu_{U \cup U}=U, \\
& \mu_{\emptyset \cup \mathcal{A}}=\mu_{\mathcal{A} \cup \emptyset}=\mu_{\mathcal{A}}, \\
\left(\mathrm{II}^{\prime}\right) . & \text { Monotonicity } \\
& {\left[\mu_{\mathcal{A}} \subseteq \mu_{\mathcal{C}}, \mu_{\mathcal{B}} \subseteq \mu_{\mathcal{D}}\right] \Longrightarrow \mu_{\mathcal{A} \cup \mathcal{B}} \subseteq \mu_{\mathcal{C} \cup \mathcal{D}}, } \\
\left(\mathrm{III}^{\prime}\right) . & \text { Commutativity } \\
& \mu_{\mathcal{A} \cup \mathcal{B}}=\mu_{\mathcal{B} \cup \mathcal{A}}, \\
\left(\mathrm{IV}^{\prime}\right) . \quad & \text { Associativity } \\
& \mu_{(\mathcal{A} \cup \mathcal{B}) \cup \mathcal{C}}=\mu_{\mathcal{A} \cup(\mathcal{B} \cup \mathcal{C})} .
\end{aligned}
$$

Compared with the quantitative conditions on t-norms and t-conorms, the qualitative properties are easier to interpret. By the boundary conditions and monotonicity, we have:

$$
\mu_{\mathcal{A} \cap \mathcal{B}} \subseteq \mu_{\mathcal{A}} \subseteq \mu_{\mathcal{A} \cup \mathcal{B}},
$$

which corresponds to the quantitative condition in equation (8).

The qualitative conditions are much weaker than the quantitative conditions. Under the qualitative conditions, fuzzy set intersection does not have to be truthfunctional. If we assume the truth-functionality of fuzzy set operators, we can obtain the quantitative properties from the qualitative ones. 
The concepts of core and support have been introduced and used as approximations of a fuzzy set [6]. The core of a fuzzy set $\mathcal{A}$ is a crisp subset of $U$ consisting of elements with full membership:

$$
\operatorname{core}\left(\mu_{\mathcal{A}}\right)=\left\{x \in U \mid \mu_{\mathcal{A}}(x)=1\right\} .
$$

The support is a crisp subset of $U$ consisting of elements with non-zero membership:

$$
\operatorname{support}\left(\mu_{\mathcal{A}}\right)=\left\{x \in U \mid \mu_{\mathcal{A}}(x)>0\right\} .
$$

With $1-(\cdot)$ as fuzzy set complement, and a pair of dual t-norm and t-conorm as fuzzy set intersection and union, the following properties hold:

$$
\begin{aligned}
& \operatorname{core}\left(\mu_{\mathcal{A}}\right)=\neg\left(\operatorname{support}\left(\mu_{\neg \mathcal{A}}\right)\right), \\
& \operatorname{support}\left(\mu_{\mathcal{A}}\right)=\neg\left(\operatorname{core}\left(\mu_{\neg \mathcal{A}}\right)\right), \\
& \operatorname{core}\left(\mu_{\mathcal{A} \cap \mathcal{B}}\right)=\operatorname{core}\left(\mu_{\mathcal{A}}\right) \cap \operatorname{core}\left(\mu_{\mathcal{B}}\right), \\
& \operatorname{support}\left(\mu_{\mathcal{A} \cap \mathcal{B}}\right) \subseteq \operatorname{support}\left(\mu_{\mathcal{A}}\right) \cap \operatorname{support}\left(\mu_{\mathcal{B}}\right), \\
& \operatorname{core}\left(\mu_{\mathcal{A} \cup \mathcal{B}}\right) \supseteq \operatorname{core}\left(\mu_{\mathcal{A}}\right) \cup \operatorname{core}\left(\mu_{\mathcal{B}}\right), \\
& \operatorname{support}\left(\mu_{\mathcal{A} \cup \mathcal{B}}\right)=\operatorname{support}\left(\mu_{\mathcal{A}}\right) \cup \operatorname{support}\left(\mu_{\mathcal{B}}\right), \\
& \operatorname{core}\left(\mu_{\mathcal{A}}\right) \subseteq \mu_{\mathcal{A}} \subseteq \operatorname{support}\left(\mu_{\mathcal{A}}\right) .
\end{aligned}
$$

Property (F1) easily follows from the definition of negation operator $n(a)=1-a$. The core of a fuzzy set is the complement of the support of the complement of the fuzzy set, and vice versa. Properties (F2) and (F3) follow from the properties of t-norm and t-conorm. The core of fuzzy set intersection can be obtained from the intersection of the cores of two fuzzy sets, and the support of fuzzy set union can be obtained from the union of the supports of two fuzzy sets. However, the support of fuzzy set intersection and the core of fuzzy set union cannot be thus obtained. Property (F4) suggests that a fuzzy set lies within its core and support.

By the boundary conditions of t-norms and t-conorms, we have:

$$
\begin{aligned}
& \left(\operatorname{support}\left(\mu_{\mathcal{A}}\right) \cap \operatorname{core}\left(\mu_{\mathcal{B}}\right)\right) \cup\left(\operatorname{core}\left(\mu_{\mathcal{A}}\right) \cap \operatorname{support}\left(\mu_{\mathcal{B}}\right)\right) \subseteq \operatorname{support}\left(\mu_{\mathcal{A} \cap \mathcal{B}}\right), \\
& \operatorname{core}\left(\mu_{\mathcal{A} \cup \mathcal{B}}\right) \subseteq\left(\operatorname{support}\left(\mu_{\mathcal{A}}\right) \cup \operatorname{core}\left(\mu_{\mathcal{B}}\right)\right) \cap\left(\operatorname{core}\left(\mu_{\mathcal{A}}\right) \cup \operatorname{support}\left(\mu_{\mathcal{B}}\right)\right) .
\end{aligned}
$$

When the min-max system, or the $\left(t_{\mathrm{p}}, s_{\mathrm{p}}\right)$ system, is used, the subset relationships become equality in (F2) and (F3), namely:

$$
\begin{array}{ll}
\left(\mathrm{F}^{\prime}\right) & \operatorname{support}\left(\mu_{\mathcal{A} \cap \mathcal{B}}\right)=\operatorname{support}\left(\mu_{\mathcal{A}}\right) \cap \operatorname{support}\left(\mu_{\mathcal{B}}\right), \\
\left(\mathrm{F} 3^{\prime}\right) & \operatorname{core}\left(\mu_{\mathcal{A} \cup \mathcal{B}}\right)=\operatorname{core}\left(\mu_{\mathcal{A}}\right) \cup \operatorname{core}\left(\mu_{\mathcal{B}}\right) .
\end{array}
$$

In order to have properties $\left(\mathrm{F} 2^{\prime}\right)$ and $\left(\mathrm{F} 3^{\prime}\right)$, we need the following additional properties on t-norms and t-conorms:

$$
\begin{array}{cc}
(\mathrm{v}) . & {[a \neq 0, b \neq 0] \Longrightarrow t(a, b) \neq 0 ;} \\
\left(\mathrm{v}^{\prime}\right) . & {[a \neq 1, b \neq 1] \Longrightarrow s(a, b) \neq 1 .}
\end{array}
$$

Unlike the standard properties of t-norms and t-conorms, they cannot be easily expressed in qualitative terms. 


\section{A Semantic Framework for Fuzzy Sets}

In the previous discussion, we focused on the qualitative properties of fuzzy set theory. Successful applications of fuzzy sets depend on, to a large extent, the interpretations of various notions of the theory. In this section, we present a semantic framework for the interpretation of fuzzy membership values, which is closely related to rough set theory [18].

When applying the theory of fuzzy sets, we do not deal with an abstract notion of sets. More often than not, the referential set $U$ contains objects relevant to a particular problem. Furthermore, we must have some information or knowledge about the objects under consideration. Such knowledge enables us to derive a more concrete and operational theory of fuzzy sets. Within the restricted theory, it is possible to discuss semantic interpretations of membership values.

In order to build a semantic framework for fuzzy sets, we consider the following two levels of knowledge about the universe:

Representations of objects: It is assumed that elements of the universe $U$ are not abstract entities, but physical objects described in terms of a set of attributes, properties or features. We perceive these objects through their features.

Relationships between objects: It is assumed that the available knowledge and information is sufficient for us to construct a certain structure on the universe $U$. That is, $U$ contains related and interconnected, rather than isolated, elements.

The two assumptions are reasonable. For solving any real world problem, we must have at least some information about the objects under consideration. We should be able to observe, measure, or describe the objects based on their properties. The fact that we consider a set of objects $U$ together implies that they must be somehow related.

At a more concrete level, we assume that each attribute has a set of values. One can observe certain relationships between attribute values. For example, one can use the trivial equality relation. From the first level knowledge, we can build the second level knowledge. The theory of rough set is developed based on the two levels of knowledge [8]. The first level of knowledge is represented by an information table. The second level of knowledge is represented by an equivalence relation on the universe. The equivalence relation on $U$ can be defined with respect to an information table.

Let $K$ represent the available information and knowledge about the universe. In contrast to the abstract fuzzy set theory, we consider the pair $(U, K)$ in an operational fuzzy set theory. The knowledge $K$ provides a basis for the definition of fuzzy sets and fuzzy set-theoretic operators. More specifically, we are interested in a constructive theory of fuzzy sets, in which fuzzy sets are explicitly constructed based on the knowledge $K$. A similar structure is used by Zhang and Zhang for the study of a granular computing approach for problem solving [25]. 
A constructive fuzzy set theory uses a triplet $(U, K, M)$, where $M$ refers to a method by which fuzzy sets are constructed. Since the construction of fuzzy sets is an inseparatable part of the theory, one can easily provide a semantic interpretation of fuzzy sets.

In the rest of this chapter, the proposed semantic framework is used to interpret fuzzy sets in rough set theory. The knowledge $K$ is given in terms of equivalence relations (partitions), non-equivalence relations, and coverings of $U$. The method $M$ is discussed according to different types of knowledge. This may provide a possible solution to a fundamental difficulty with fuzzy set theory regarding semantic interpretations of fuzzy membership values.

\section{Classical Rough Membership Functions and Rough Set Approximations}

The notion of indiscernibility provides a formal way to describe the relationships between elements of a universe. Two elements are said to be indiscernible, indistinguishable, or equivalent if one cannot differentiate them based on the available information or knowledge. The theory of rough sets is a model of uncertainty developed based on the notion of indiscernibility $[7,14,16]$.

\subsection{Rough membership functions}

Let $E \subseteq U \times U$ be an equivalence relation on a finite and non-empty universe $U$. That is, $E$ is reflexive, symmetric, and transitive. The relation $E$ induces a partition $U / E$ of the universe $U$, i.e., a family of disjoint subsets of the universe known as equivalence classes. The equivalence relation represents the available knowledge about the universe. In the presence of indiscernibility, we are forced to consider an equivalence class as a whole instead of individuals. It is therefore expected that any model of uncertainty should take into consideration the equivalence or indiscernibility of elements.

The pair, $a p r=(U, E)$, is called an approximation space. An element $x \in U$ belongs to one and only one equivalence class. Let

$$
[x]_{E}=\{y \mid x E y\}
$$

denote the equivalence class containing $x$. For a subset $A \subseteq U$, we define a rough membership function $[9,11,21]$ :

$$
\mu_{A}(x)=\frac{\left|[x]_{E} \cap A\right|}{\left|[x]_{E}\right|},
$$

where $|\cdot|$ denotes the cardinality of a set. The rough membership value $\mu_{A}(x)$ may be interpreted as the conditional probability that an arbitrary element belongs to $A$ given that the element belongs to $[x]_{E}$. The set $A$ is called a generating set of the rough membership $\mu_{A}$. 
Rough membership functions may be interpreted as fuzzy membership functions in terms of the probabilities defined simply by the cardinalities of sets. With this interpretation, one can define at most $2^{|U|}$ fuzzy sets. Two distinct subsets of $U$ may derive the same rough membership function. By definition, the membership values are all rational numbers.

The theory of fuzzy sets is typically developed as an uninterpreted mathematical theory of abstract membership functions without the above limitations [6]. In contrast, the theory of rough set provides a more specific and more concrete interpretation of fuzzy membership functions. The source of the fuzziness in describing a concept is the indiscernibility of elements. The limitations and constraints of such an interpreted sub-theory should not be viewed as the disadvantages of the theory. In fact, such constraints suggest conditions that can be verified when applying the theory to real world problems. It might be more instructive and informative if one knows that a certain theory cannot be applied. Explicit statements of conditions under which a particular model is applicable may prevent misuses of the theory.

When interpreting fuzzy membership functions in the theory of rough sets, we have the constraints:

$$
\begin{array}{ll}
(\mathrm{rm} 1) & \mu_{U}(x)=1, \\
(\mathrm{rm} 2) & \mu_{\emptyset}(x)=0, \\
(\mathrm{rm} 3) & x E y \Longrightarrow \mu_{A}(x)=\mu_{A}(y), \\
(\mathrm{rm} 4) & x \in A \Longrightarrow \mu_{A}(x) \neq 0 \\
& \mu_{A}(x)=0 \Longrightarrow x \notin A, \\
(\mathrm{rm} 5) & \mu_{A}(x)=1 \Longrightarrow x \in A, \\
& x \notin A \Longrightarrow \mu_{A}(x) \neq 1, \\
(\operatorname{rm} 6) & A \subseteq B \Longrightarrow \mu_{A}(x) \leq \mu_{B}(x) .
\end{array}
$$

Property (rm3) is particularly important. It shows one type of constraints on membership values. Elements in the same equivalence class must have the same degree of membership. That is, indiscernible elements should have the same membership value. Such a constraint, which ties the membership values of individual elements according to their connections, is intuitively appealing. The two properties in (rm4) are equivalent. An element in $A$ must have a non-zero membership, while an element with zero membership cannot be in $A$. Similarly, properties in (rm5) state that an element with full membership must be in $A$, and an element not in $A$ cannot have a full membership. Property (rm6) shows another type of constraints on membership values. A subset of a set generates a fuzzy subset of the corresponding fuzzy set generated by that set.

The constraints on rough membership functions have significant implications on rough set-theoretic operators. Rough membership functions corresponding to $\neg A, A \cap B$, and $A \cup B$ must be defined using set-theoretic operators and equation (14). By laws of probability, we have:

$$
\mu_{\neg A}(x)=1-\mu_{A}(x),
$$




$$
\mu_{A \cup B}(x)=\mu_{A}(x)+\mu_{B}(x)-\mu_{A \cap B}(x),
$$

$$
\begin{aligned}
& A \cap B=\emptyset \Longrightarrow \mu_{A \cup B}(x)=\mu_{A}(x)+\mu_{B}(x), \\
& \max \left(0, \mu_{A}(x)+\mu_{B}(x)-1\right) \leq \mu_{A \cap B}(x) \leq \min \left(\mu_{A}(x), \mu_{B}(x)\right),
\end{aligned}
$$

$$
\max \left(\mu_{A}(x), \mu_{B}(x)\right) \leq \mu_{A \cup B}(x) \leq \min \left(1, \mu_{A}(x)+\mu_{B}(x)\right) .
$$

Unlike the commonly used fuzzy set operators as typically defined by t-norms and t-conorms, the new intersection and union operators are non-truth-functional. That is, it is impossible to obtain rough membership functions of $A \cap B$ and $A \cup B$ based solely on the rough membership functions of $A$ and $B$. One must also consider their overlap and their relationships to the equivalence class $[x]_{E}$.

By property (o2), we have the following formula to calculate the membership function of a set:

$$
\mu_{A}(x)=\sum_{y \in A} \mu_{\{y\}}(x) .
$$

Thus, rough membership functions corresponding to singleton subsets of $U$ play an important role. They serve as a basis of rough membership functions generated by non-singleton subsets.

\subsection{Rough set approximations and approximation operators}

In an approximation space, a subset $A \subseteq U$ is approximated by a pair of sets called the lower and upper approximations [9]:

$$
\begin{aligned}
\underline{\operatorname{apr}}(A) & =\left\{x \in U \mid \mu_{A}(x)=1\right\} \\
& =\operatorname{core}\left(\mu_{A}\right), \\
\overline{\operatorname{apr}}(A) & =\left\{x \in U \mid \mu_{A}(x)>0\right\} \\
& =\operatorname{support}\left(\mu_{A}\right) .
\end{aligned}
$$

They are indeed the core and support of the fuzzy set $\mu_{A}$.

The lower and upper approximations may be viewed as two operators from $2^{U}$ to $2^{U}$, where $2^{U}$ is the power set of $U$. The system $\left(2^{U}, \neg, \cap, \cup, a p r, \overline{a p r}\right)$ is called a rough set algebra [13]. It may be considered as the Boolean algebra $\left(2^{U}, \neg, \cap, \cup\right)$ with two added unary operators $\underline{a p r}$ and $\overline{a p r}$. One can verify that the approximation operators satisfy the following conditions: for subsets $A, B \subseteq$ $U$,

$(\mathrm{R} 1)$

$$
\begin{aligned}
& \underline{\operatorname{apr}}(A)=\neg(\overline{\operatorname{apr}}(\neg A)), \\
& \overline{\operatorname{apr}}(A)=\neg \underline{\underline{\operatorname{apr}}(\neg A)),}
\end{aligned}
$$

$$
\underline{a p r}(A \cap B)=\underline{\operatorname{apr}}(A) \cap \underline{\operatorname{apr}}(B),
$$$$
\overline{\overline{a p r}}(A \cap B) \subseteq \overline{\overline{a p r}}(A) \cap \overline{\overline{a p r}}(B),
$$

$$
\begin{aligned}
& \underline{\operatorname{apr}}(A \cup B) \supseteq \underline{a p r}(A) \cup \underline{\operatorname{apr}}(B), \\
& \overline{\overline{a p r}}(A \cup B)=\overline{\overline{a p r}}(A) \cup \overline{\overline{a p r}}(B),
\end{aligned}
$$

$$
\underline{a p r}(A) \subseteq A \subseteq \overline{a p r}(A)
$$


Property (R1) suggests that approximation operators are a pair of dual operators. By (R2) and (R3), the lower approximation operator is distributive over set intersection, and the upper approximation operators is distributive over set union. By comparing with (F1)-(F4), we can see that rough set approximation operators satisfy the same properties of core and support of fuzzy sets. In other words, fuzzy sets in general and fuzzy sets defined in rough set theory share the same qualitative characteristics.

Rough set approximations can be re-expressed in the following equivalent forms:

$$
\begin{aligned}
\underline{\operatorname{apr}(A)} & =\left\{x \in U \mid[x]_{E} \subseteq A\right\} \\
& =\{x \in U \mid \forall y \in U(x E y \Longrightarrow y \in A)\}, \\
& =\bigcup\left\{[x]_{E} \mid[x]_{E} \subseteq A\right\} \\
\overline{\operatorname{apr}}(A) & =\left\{x \in U \mid[x]_{E} \cap A \neq \emptyset\right\} \\
& =\{x \in U \mid \exists y \in U(x E y, y \in A)\} \\
& =\bigcup\left\{[x]_{E} \mid[x]_{E} \cap A \neq \emptyset\right\} .
\end{aligned}
$$

They represent two additional definitions of rough set approximations [19]. The element based definition focuses on single elements of $U$. An element $x \in U$ is in the lower approximation, i.e., $\mu_{A}(x)=1$, if and only if for all $y \in U$, $x E y$ implies $y \in A$. An element $x \in U$ is in the upper approximation, i.e., $\mu_{A}(x) \neq 0$, if and only if there exists a $y \in U$ such that $x E y$ and $y \in A$. This definition clearly relates rough set theory to modal logic [20]. It is useful in the generalization of rough sets using non-equivalence relations. On the other hand, the equivalence class (granule) based definition focuses on equivalence classes of the partition $U / E$. The lower approximation $\operatorname{apr}(A)$ is the union of those equivalence classes which are subsets of $A$. The upper approximation $\overline{\operatorname{apr}}(A)$ is the union of those equivalence classes which have non-empty intersections with $A$. This definition is useful when generalizing rough sets using coverings of the universe. The equivalence of the two definitions can be seen from property ( $\mathrm{rm} 3$ ), namely, elements in the same equivalence class have the same membership.

Rough membership functions describe a particular type of fuzziness induced by indiscernibility of elements. In an approximation space $a p r=(U, E)$, a set is referred to as non-fuzzy if it generates a crisp subset of $U$. More specifically, a set is non-fuzzy if $\operatorname{apr}(A)=A=\overline{a p r}(A)$. In other words, a subset $A$ of $U$ is non-fuzzy if the indiscernibility does not lead to fuzziness in our perception of $A$. The empty set, the equivalence classes, and unions of equivalence classes are non-fuzzy. The set of non-fuzzy sets, denoted by $\operatorname{Def}(U)$, consists of $\emptyset$ and $U$, and is closed under $\neg, \cap$ and $\cup$. That is, $\operatorname{Def}(U)$ is a sub-Boolean algebra of $2^{U}$. All other subsets of $U$ induce non-crisp fuzzy sets. By the equivalence class based definition, both $\mu_{\underline{a p r}(A)}$ and $\mu_{\overline{a p r}(A)}$ are crisp sets $\underline{\operatorname{apr}}(A)$ and $\overline{\operatorname{apr}}(A)$. 


\subsection{Main features of the rough set based semantics of fuzzy sets}

From the proposed semantic interpretation of fuzzy sets in the theory of rough sets, one can observe several important features. They are important for understanding the semantics of fuzzy sets. They are summarized below.

Fuzzy sets as a derived notion. Fuzzy sets are not treated as the primitive notion, but a derived notion. In particular, a fuzzy set is generated by a crisp set using an equivalence relation. By treating fuzzy sets as a derived notion, one is able to develop different semantics of fuzzy sets. Other primitive notions, which have sound semantic interpretations, can be used to define fuzzy sets. In rough set theory, the notion of equivalence relations can be easily defined and explained using an information table [8].

Representation of the source of fuzziness. The indiscernibility, represented by an equivalence relation, is the source of fuzziness in rough set theory. With respect to indiscernibility, some subsets of the universe cannot be precisely described, and are consequently described by fuzzy subsets. By explicitly defining the source and the form of fuzziness, we have a clear understanding of the meaning of fuzzy sets. Furthermore, even under the indiscernibility of elements, certain subsets of the universe are non-fuzzy. The distinction made between fuzzy and non-fuzzy subsets may provide more insights to the semantics of fuzzy sets.

Qualitative representation of fuzzy sets. The core and support are qualitative representations of a fuzzy set. In rough set theory, the core and support are referred to as the lower and upper approximations of a set. Although fuzzy sets defined in rough set theory are a special class of fuzzy sets, they share the same qualitative properties of any fuzzy set system defined by a pair of dual t-norm and t-conorm. This implies that the semantics of fuzzy sets presented here is not as restricted as it appears.

Constraints on fuzzy membership functions. The proposed semantics of fuzzy sets clearly shows two types of constraints on fuzzy membership functions. One type of constraints suggests that the membership values of two related elements are related. The other type of constraints suggests that fuzzy membership functions generated from two related sets (concepts) should be related. It should also be pointed out that the constraints on fuzzy membership functions are of a qualitative nature.

Constraints on fuzzy set-theoretic operators. Within rough set theory, there are constraints on fuzzy set-theoretic operators. It is clearly shown that fuzzy set-theoretic operators are not truth-functional.

Our discussions rely on a qualitative characterization of fuzzy sets. Rough set approximations are core and support of fuzzy sets. The t-norms and t-conorms deal with qualitative requirements on fuzzy set-theoretic operators. The qualitative semantic interpretation of fuzzy sets may provide more insights into the theory of fuzzy sets. 


\section{Generalized Rough Sets by Non-equivalence Relations}

Standard rough sets are defined based on an equivalence relation, i.e., a reflexive, symmetric and transitive relation, on a finite and non-empty universe [7]. Yao et al. considered generalized rough sets based on an arbitrary binary relation [13, $20]$.

\subsection{Binary relations}

Let $R \subseteq U \times U$ be a binary relation on a finite and non-empty universe $U$. For two elements $x, y \in U$, if $x R y$, we say that $x$ is $R$-related to $y$. Let

$$
\begin{aligned}
& R_{s}(x)=\{y \in U \mid x R y\} \\
& R_{p}(x)=\{y \in U \mid y R x\}
\end{aligned}
$$

denote the successor and the predecessor neighborhoods of $x$ induced by the binary relation $R$. Additional neighborhoods defined by a binary relation have also been studied $[12,17]$.

Different classes of binary relations can be obtained by considering the following properties:

$$
\begin{array}{ll}
\text { serial : } & \forall x \in U \exists y \in U[x R y], \\
& \forall x \in U\left[R_{s}(x) \neq \emptyset\right], \\
\text { inverse serial : } \forall x \in U \exists y \in U[y R x], & \\
& \forall x \in U\left[R_{p}(x) \neq \emptyset\right], \\
\text { reflexive : } \quad & \forall x \in U[x R x], \\
& \forall x \in U\left[x \in R_{s}(x)\right], \\
\text { symmetric : } & \forall x, y \in U[x R y \Longrightarrow y R x], \\
& \forall x, y \in U\left[y \in R_{s}(x) \Longrightarrow x \in R_{s}(y)\right], \\
\text { transitive : } & \forall x, y, z \in U[(x R y, y R z) \Longrightarrow x R z], \\
& \forall x, y \in U\left[y \in R_{s}(x) \Longrightarrow R_{s}(y) \subseteq R_{s}(x)\right], \\
\text { Euclidean : } & \forall x, y, z \in U[(x R y, x R z) \Longrightarrow y R z], \\
& \forall x, y \in U\left[y \in R_{s}(x) \Longrightarrow R_{s}(x) \subseteq R_{s}(y)\right] .
\end{array}
$$

A relation is called a tolerance (compatibility) relation if it is reflexive and symmetric.

From a binary relation $R$, we can define four binary relations:

$$
\begin{aligned}
& x \equiv_{R} y \Longleftrightarrow R_{s}(x)=R_{s}(y), \\
& x \approx_{R} y \Longleftrightarrow R_{s}(x) \cap R_{s}(y) \neq \emptyset \\
& x \simeq_{R} y \Longleftrightarrow R_{p}(x)=R_{p}(y), \\
& x \sim_{R} y \Longleftrightarrow R_{p}(x) \cap R_{p}(y) \neq \emptyset .
\end{aligned}
$$

Relations $\equiv_{R}$ and $\simeq_{R}$ are reflexive, symmetric, and transitive, and hence are equivalence relations. Relations $\approx_{R}$ and $\sim_{R}$ are symmetric. If $R$ is a serial relation, then $\approx_{R}$ is also reflexive, and hence a tolerance relation. If $R$ is inverse 
serial, $\sim_{R}$ is reflexive, and hence a tolerance relation. While $\equiv_{R}$ and $\simeq_{R}$ show strong connections between elements of $U, \approx_{R}$ and $\sim_{R}$ show some weak connections. Two elements are considered to be equivalent if they have the same successor (predecessor) neighborhood, and similar if their successor (predecessor) neighborhoods have a non-empty overlap.

\subsection{Rough membership functions}

The pair apr $=(U, R)$ is called an approximation space, with the relation $R$ representing the relationships between elements of $U$. One can define rough membership functions by extending equation (14) using neighborhoods induced by a binary relation [14]. We consider the definition based on the successor neighborhoods. For a subset $A \subseteq U$, a rough membership function can be defined by substituting $[x]_{E}$ with $R_{s}(x)$ in equation (14) as follows:

$$
\mu_{A}(x)=\frac{\left|R_{s}(x) \cap A\right|}{\left|R_{s}(x)\right|} .
$$

We assume that relation $R$ is at least serial, i.e., $R_{s}(x) \neq \emptyset$ for all $x \in U$. Under this assumption, the rough membership function is well defined.

Recall that we can identify at least two types of constraints. One type suggests that membership values of related elements should be related. Another type suggests that two rough membership functions obtained from two related generating sets should be related. It is naturally expected that relationships given by binary relations should impose some constraints on rough membership functions.

For an arbitrary binary relation $R$, we have:

$$
\text { (I) } \quad x R y \Longleftrightarrow \mu_{\{y\}}(x) \neq 0 .
$$

If the relation $R$ is reflexive, the following condition holds:

$$
\mu_{R_{s}(x)}(x)=1 .
$$

They serve as basic properties for us to derive other properties of rough membership functions. Suppose the relation $R$ is both serial and inverse serial. The rough membership functions still satisfy ( $\mathrm{rm} 1)$, (rm2), and (rm6). Corresponding to property $(\mathrm{rm} 3)$, we have the following constraints:

$$
\begin{array}{ll}
(\operatorname{rm} 3.1) & x \equiv_{R} y \Longrightarrow \mu_{A}(x)=\mu_{A}(y) \\
(\operatorname{rm} 3.2) & \left(\mu_{A}(x)=1, x \approx_{R} y\right) \Longrightarrow \mu_{A}(y) \neq 0, \\
& \left(\mu_{A}(x)=0, x \approx_{R} y\right) \Longrightarrow \mu_{A}(y) \neq 1, \\
(\operatorname{rm} 3.3) & x \simeq_{R} y \Longrightarrow \mu_{\{x\}}(z)=\mu_{\{y\}}(z), \\
(\operatorname{rm} 3.4) & \left(\mu_{\{x\}}(z) \neq 0, \neg\left(x \sim_{R} y\right)\right) \Longrightarrow \mu_{\{y\}}(z)=0 .
\end{array}
$$

The first two properties state one type of constraints, namely, membership values of related elements are related. According to ( $\mathrm{rm} 3.1)$, if two elements are 
equivalent, in the sense defined by $\equiv_{R}$, then they must have the same membership value. According to ( $\mathrm{rm} 3.2)$, if an element $y$ is similar to another element $x$ with full membership, in the sense defined by $\approx_{R}$, then $y$ cannot have a null membership. Likewise, if $y$ is similar to an element $x$ with null membership, then $y$ cannot have a full membership. The last two properties show another type of constraints, namely, rough membership functions derived from two related generating sets must be related. By ( $\mathrm{rm} 3.3$ ), if $x$ and $y$ are equivalent, in the sense of $\simeq_{R}$, we can conclude that $\{x\}$ and $\{y\}$ generate the same rough membership function. By (rm3.4), if $z$ has a non-zero membership value in $\mu_{\{x\}}$ and $\neg\left(x \sim_{R} y\right)$, we can conclude that $z$ must have a zero membership value in $\mu_{\{y\}}$. That is, if $x$ is not similar to $y, x$ and $y$ generate totally different rough membership functions in the sense that whenever $z$ has a non-zero membership value for one, it must have the zero membership value for the other.

Rough membership values defined using an arbitrary binary relation are also conditional probabilities. Consequently, properties (o1)-(o4) hold for rough set operators.

\subsection{Rough set approximation and approximation operators}

Similar to the classical case, a pair of rough set approximations can be defined by the core and support of $\mu_{A}$. That is, for a set $A \subseteq U$, we have [13,20]:

$$
\begin{aligned}
\underline{\operatorname{apr}(A)} & =\operatorname{core}\left(\mu_{A}\right), \\
& =\left\{x \in U \mid \mu_{A}(x)=1\right\} \\
& =\left\{x \in U \mid R_{s}(x) \subseteq A\right\} \\
& =\{x \in U \mid \forall y \in U(x R y \Longrightarrow y \in A)\}, \\
\overline{\operatorname{apr}}(A) & =\operatorname{support}\left(\mu_{A}\right) \\
& =\left\{x \in U \mid \mu_{A}(x)>0\right\} \\
& =\left\{x \in U \mid R_{s}(x) \cap A \neq \emptyset\right\} \\
& =\{x \in U \mid \exists y \in U(x R y, y \in A)\} .
\end{aligned}
$$

Independent of the properties of the binary relation, they satisfy properties (R1)(R3).

Properties of a binary relation determine the properties of approximation operators $a p r$ and $\overline{a p r}$. With respect to the serial, reflexive, symmetric, transitive and Euclidean properties, the approximation operators have the corresponding properties:

$$
\begin{array}{ll}
\text { (D) } & \underline{\operatorname{apr}}(A) \subseteq \overline{\operatorname{apr}}(A) ; \\
\text { (T) } & \overline{\operatorname{apr}}(A) \subseteq A, \\
& A \subseteq \overline{\operatorname{apr}}(A) ; \\
\text { (B) } & A \subseteq \underline{\operatorname{apr}}(\overline{\operatorname{apr}}(A)), \\
& \overline{a p r}(\underline{\operatorname{apr}}(A)) \subseteq A ;
\end{array}
$$




$$
\begin{aligned}
& \underline{\operatorname{apr}}(A) \subseteq \underline{\operatorname{apr}}(\operatorname{apr}(A)), \\
& \overline{\operatorname{apr}}(\overline{\operatorname{apr}}(A)) \subseteq \overline{\operatorname{apr}}(A) ; \\
& \overline{\operatorname{apr}}(A) \subseteq \underline{\operatorname{apr}}(\overline{\operatorname{apr}}(A)), \\
& \overline{\operatorname{apr}}(\underline{\operatorname{apr}}(A)) \subseteq \underline{\operatorname{apr}}(A) .
\end{aligned}
$$

By combining properties of binary relations, we can produce more classes of rough set models.

\subsection{Classes of rough set models}

With respect to properties of a binary relation, such as reflexive, symmetric, transitive, and Euclidean, we can identify additional constraints on rough membership functions. For simplicity, we only consider constraints on membership values with respect to the same fuzzy set. In this case, they are the re-expression of properties (T), (B), (4) and (5) in terms of rough membership functions. Thus, we use the same symbols to label the corresponding properties.

Reflexive model A reflexive relation is a special serial relation. In this case, for all $x \in U, x R x$ or equivalently $x \in R_{s}(x)$. The corresponding equivalent properties of rough membership functions can be stated as:

$$
\text { (T) } \quad \begin{array}{ll} 
& \mu_{\{x\}}(x) \neq 0 ; \\
& x \in A \Longrightarrow \mu_{A}(x) \neq 0 ; \\
& \mu_{A}(x)=1 \Longrightarrow x \in A .
\end{array}
$$

They are in fact the properties ( $\mathrm{rm} 4)$ and (rm5). The equivalence of the properties can be easily shown. As an example, we show the equivalence of the first and the last properties. Assume $\mu_{\{x\}}(x) \neq 0$. By (I), we can conclude that $x \in R_{s}(x)$. If $\mu_{A}(x)=1$, we have $R_{s}(x) \subseteq A$. By combining $x \in R_{s}(x)$ and $R_{s}(x) \subseteq A$, we have $x \in A$. This means that the first property implies the last property. Now assume $\mu_{A}(x)=1 \Longrightarrow x \in A$. According to (II), we have $\mu_{R_{s}(x)}(x)=1$. Thus, $x \in R_{s}(x)$, which implies $\mu_{\{x\}}(x) \neq 0$. We have therefore showed that the last property implies the first property.

Symmetric model For a symmetric relation, if $x \in R_{s}(y)$ then $y \in R_{s}(x)$, namely, $R_{p}(x)=R_{s}(x)$. Relations $\equiv_{R}$ and $\simeq_{R}$ become the same, and relations $\approx_{R}$ and $\sim_{R}$ become the same. In a symmetric model, we have the following equivalent properties on a rough membership function:

(B)

$$
\begin{aligned}
& \mu_{\{y\}}(x) \neq 0 \Longrightarrow \mu_{\{x\}}(y) \neq 0 ; \\
& {[x \in A, x R y] \Longrightarrow \mu_{A}(y) \neq 0 ;} \\
& {\left[\mu_{A}(y)=0, x R y\right] \Longrightarrow x \notin A ;} \\
& {\left[\mu_{A}(y)=1, x R y\right] \Longrightarrow x \in A ;} \\
& {[x \notin A, x R y] \Longrightarrow \mu_{A}(y) \neq 1 .}
\end{aligned}
$$


These properties are similar, in form, to properties (rm3.2) and (rm3.4). Furthermore, they also contain conditions in (T). Property (B) states constraints on the membership functions directly through the binary relation $R$. For example, if $y$ is $R$-related to an element $x \in A$, then $y$ has a non-zero membership value in $\mu_{A}$. On the other hand, (rm3.2) and (rm3.4) state constraints indirectly through $R$.

Transitive model For a transitive relation $R$, for all $x, y \in U$, if $x R y$, then $R_{s}(y) \subseteq R_{s}(x)$. For a transitive relation $R$, we have the connection between $R$ and $\approx_{R}$ and $\sim_{R}$ :

$$
\begin{aligned}
& R \text { is serial and transitive } \Longrightarrow\left[x R y \Longrightarrow x \approx_{R} y\right] ; \\
& R \text { is inverse serial and transitive } \Longrightarrow\left[x R y \Longrightarrow x \sim_{R} y\right] .
\end{aligned}
$$

In a transitive model, we have the following equivalent properties on membership functions:

$$
\text { (4) } \begin{aligned}
& \mu_{\{y\}}(x) \neq 0 \Longrightarrow \mu_{R_{s}(x)}(y)=1 ; \\
& {\left[\mu_{A}(x)=1, x R y\right] \Longrightarrow \mu_{A}(y)=1 ;} \\
& {\left[\mu_{A}(y) \neq 1, x R y\right] \Longrightarrow \mu_{A}(x) \neq 1 ;} \\
& {\left[\mu_{A}(x) \neq 0, y R x\right] \Longrightarrow \mu_{A}(y) \neq 0 ;} \\
& {\left[\mu_{A}(y)=0, y R x\right] \Longrightarrow \mu_{A}(x)=0 .}
\end{aligned}
$$

Combining the above results with (rm3.2) and (rm3.4), we can obtain additional properties.

Euclidean model For an Euclidean relation, for all $x, y \in U$, if $x R y$, then $R_{s}(x) \subseteq R_{s}(y)$. In this case, we have:

$$
\begin{aligned}
& R \text { is Euclidean } \Longrightarrow\left[x R y \Longrightarrow x \approx_{R} y\right] ; \\
& R \text { is inverse serial and Euclidean } \Longrightarrow\left[x R y \Longrightarrow x \sim_{R} y\right] .
\end{aligned}
$$

The following properties can be obtained:

$$
\begin{aligned}
& \mu_{\{y\}}(x) \neq 0 \Longrightarrow \mu_{R_{s}(y)}(x)=1 ; \\
& {\left[\mu_{A}(x) \neq 0, x R y\right] \Longrightarrow \mu_{A}(y) \neq 0 ;} \\
& {\left[\mu_{A}(y)=0, x R y\right] \Longrightarrow \mu_{A}(x)=0 ;} \\
& {\left[\mu_{A}(x)=1, y R x\right] \Longrightarrow \mu_{A}(y)=1 ;} \\
& {\left[\mu_{A}(y) \neq 1, y R x\right] \Longrightarrow \mu_{A}(x) \neq 1 .}
\end{aligned}
$$

They can be used to infer additional properties.

Pawlak model A Pawlak model is characterized by an equivalence relation. In this case, all four relations $\equiv_{R}, \approx_{R}, \simeq_{R}$ and $\sim_{R}$ are the same as the relation $R$. An equivalence relation is an Euclidean relation. Thus, all the properties established so far hold for a Pawlak model. 


\section{Generalized Rough Sets based on Coverings of the Universe}

In a partition, an element belongs to one equivalence class and two distinct equivalence classes have no overlap. Covering of the universe is a natural generalization of partitions, in which an element can be in more than one class of a covering. This provides a more realistic model for the formulation of rough sets $[14,24]$.

\subsection{Coverings}

A covering of the universe, $\mathbf{C}=\left\{C_{1}, \ldots, C_{n}\right\}$, is a family of subsets of $U$ such that $U=\bigcup\left\{C_{i} \mid i=1, \ldots, n\right\}$. Two distinct sets in $\mathbf{C}$ may have a non-empty overlap. An element may belong to more than one class in $\mathbf{C}$. The family $\mathbf{C}(x)=$ $\{C \in \mathbf{C} \mid x \in C\}$ consists of sets in $\mathbf{C}$ containing $x$. With respect to a covering, we construct the following equivalence relation:

$$
\begin{aligned}
x \equiv_{\mathbf{C}} y & \Longleftrightarrow \forall C \in \mathbf{C}(x \in C \Longleftrightarrow y \in C) \\
& \Longleftrightarrow \mathbf{C}(x)=\mathbf{C}(y) .
\end{aligned}
$$

Two elements are considered to be equivalent if they appear in the same family of subsets in $\mathbf{C}$. A tolerance relation $\sim_{\mathbf{C}}$ can be defined by:

$$
\begin{aligned}
x \sim_{\mathbf{C}} y & \Longleftrightarrow \exists C \in \mathbf{C}(x \in C, y \in C) \\
& \Longleftrightarrow \mathbf{C}(x) \cap \mathbf{C}(y) \neq \emptyset .
\end{aligned}
$$

That is, $x$ and $y$ are considered to be similar if they appear together in at least one class of the covering.

\subsection{Rough membership functions}

The sets in $\mathbf{C}(x)$ describe different types or various degrees of similarity between elements of $U$. For a set $C \in \mathbf{C}(x)$, we may compute a value $|C \cap A| /|C|$ by extending equation (14). It may be interpreted as the membership value of $x$ from the view point of $C$. With respect to a covering $\mathbf{C}$, we have a family of values $\{|C \cap A| /|C| \mid x \in C, C \in \mathbf{C}\}$. Generalized rough membership functions may be defined by using this family of values. We consider the following three definitions [22]:

$$
\begin{aligned}
\text { (minimum) } & \underline{\mu}_{A}(x)=\min \left\{\frac{|C \cap A|}{|C|} \mid x \in C, C \in \mathbf{C}\right\}, \\
\text { (maximum) } & \bar{\mu}_{A}(x)=\max \left\{\frac{|C \cap A|}{|C|} \mid x \in C, C \in \mathbf{C}\right\}, \\
\text { (average) } & \mu_{A}^{*}(x)=\operatorname{avg}\left\{\frac{\left|C_{i} \cap A\right|}{\left|C_{i}\right|} \mid x \in C, C \in \mathbf{C}\right\} .
\end{aligned}
$$


The minimum, maximum, and average definitions may be regarded as the most permissive, the most optimistic, and a balanced view, respectively, in defining rough membership functions. The minimum rough membership function is determined by a set in $\mathbf{C}(x)$ which has the smallest overlap with $A$, and the maximum rough membership function by a set in $\mathbf{C}(x)$ which has the largest overlap with $A$. The average rough membership function depends on every set in $\mathbf{C}(x)$.

The generalized rough membership functions have the following properties:

$$
\begin{array}{ll}
(\operatorname{grm} 0) & \underline{\mu}_{A}(x) \leq \mu_{A}^{*}(x) \leq \bar{\mu}_{A}(x) \\
(\operatorname{grm} 1) & \underline{\mu}_{U}(x)=\mu_{U}^{*}(x)=\bar{\mu}_{U}(x)=1 \\
(\operatorname{grm} 2) & \underline{\mu}_{\emptyset}(x)=\mu_{\emptyset}^{*}(x)=\bar{\mu}_{\emptyset}(x)=0 \\
(\operatorname{grm} 3) & x \equiv_{\mathbf{C}} y \Longrightarrow\left[\underline{\mu}_{A}(x)=\underline{\mu}_{A}(y), \mu_{A}^{*}(x)=\mu_{A}^{*}(y), \bar{\mu}_{A}(x)=\bar{\mu}_{A}(y)\right] \\
(\operatorname{grm} 4) & \left(x \sim_{\mathbf{C}} y, \underline{\mu}_{A}(x)=1\right) \Longrightarrow \bar{\mu}_{A}(y)=1 \\
& \left(x \sim_{\mathbf{C}} y, \bar{\mu}_{A}(x)=0\right) \Longrightarrow \underline{\mu}_{A}(y)=0 \\
& \left(x \sim_{\mathbf{C}} y, \bar{\mu}_{A}(x)=0\right) \Longrightarrow \bar{\mu}_{A}^{*}(y) \neq 1 \\
& \left(x \sim_{\mathbf{C}} y, \underline{\mu}_{A}(x)=1\right) \Longrightarrow \mu_{A}^{*}(y) \neq 0 \\
(\operatorname{grm} 5) & x \in A \Longrightarrow \underline{\mu}_{A}(x) \neq 0 \\
& \underline{\mu}_{A}(x)=0 \Longrightarrow x \notin A \\
& x \in A \Longrightarrow \bar{\mu}_{A}(x) \neq 0 \\
& \bar{\mu}_{A}(x)=0 \Longrightarrow x \notin A \\
(\operatorname{grm} 6) & \bar{\mu}_{A}(x)=1 \Longrightarrow x \in A \\
& x \notin A \Longrightarrow \bar{\mu}_{A}(x) \neq 1 \\
& \underline{\mu}_{A}(x)=1 \Longrightarrow x \in A \\
& x \notin A \Longrightarrow \underline{\mu}_{A}(x) \neq 1 \\
(\operatorname{grm} 7) \quad & A \subseteq B \Longrightarrow \underline{\mu}_{A}(x) \leq \underline{\mu}_{B}(x) \\
& A \subseteq B \Longrightarrow \bar{\mu}_{A}(x) \leq \bar{\mu}_{B}(x)
\end{array}
$$

Property (grm0) states the relationship between three membership functions. A partition is a special type of covering. In this case, three rough membership functions reduce to the same rough membership function. Properties (grm1) and (grm2) show the membership values of two special sets, the empty set $\emptyset$ and the entire set $U$. Both (grm3) and (grm4) show the constraints on rough membership functions imposed by the similarity of objects. From the relation $\underline{\mu}_{A}(x) \leq \mu_{A}^{*}(x) \leq \bar{\mu}_{A}(x)$, we can obtain additional properties. For example, (grm5) implies that $x \in A \Longrightarrow \mu_{A}^{*}(x) \neq 0$. Similarly, (grm6) implies that $\mu_{A}^{*}(x)=1 \Longrightarrow x \in A$.

For set-theoretic operators, one can verify the following properties:

$$
\begin{aligned}
& \underline{\mu}_{\neg A}(x)=1-\bar{\mu}_{A}(x), \\
& \bar{\mu}_{\neg A}(x)=1-\underline{\mu}_{A}(x), \\
& \mu_{\neg A}^{*}(x)=1-\mu_{A}^{*}(x), \\
& \max \left(0, \underline{\mu}_{A}(x)+\underline{\mu}_{B}(x)-\bar{\mu}_{A \cup B}(x)\right) \leq \underline{\mu}_{A \cap B}(x) \leq \min \left(\underline{\mu}_{A}(x), \underline{\mu}_{B}(x)\right),
\end{aligned}
$$




$$
\begin{aligned}
& \max \left(\bar{\mu}_{A}(x), \bar{\mu}_{B}(x)\right) \leq \bar{\mu}_{A \cup B}(x) \leq \min \left(1, \bar{\mu}_{A}(x)+\bar{\mu}_{B}(x)-\underline{\mu}_{A \cap B}(x)\right), \\
& \mu_{A \cap B}^{*}(x)=\mu_{A}^{*}(x)+\mu_{B}^{*}(x)-\mu_{A \cup B}^{*}(x) .
\end{aligned}
$$

We again obtain non-truth-functional rough set operators.

The minimum rough membership function may be viewed as the lower bound on all possible rough membership functions definable using a covering, while the maximum rough membership as the upper bound. The pair $\left(\underline{\mu}_{A}(x), \bar{\mu}_{A}(x)\right)$ may also be used to define an interval-valued fuzzy set [6]. The interval $\left[\underline{\mu}_{A}(x), \bar{\mu}_{A}(x)\right]$ is the membership value of $x$ with respect to $A$.

\subsection{Rough set approximations and approximation operators}

From the three rough membership functions, we define three pairs of lower and upper approximations. For the minimum definition, we have:

$$
\begin{aligned}
\underline{a p r}^{m}(A) & =\operatorname{core}\left(\underline{\mu}_{A}\right) \\
& =\left\{x \in U \mid \underline{\mu}_{A}(x)=1\right\} \\
& =\{x \in U \mid \forall C \in \mathbf{C}(x \in C \Longrightarrow C \subseteq A)\}, \\
\overline{a p r}^{m}(A) & =\operatorname{support}\left(\underline{\mu}_{A}\right) \\
& =\left\{x \in U \mid \underline{\mu}_{A}(x)>0\right\} \\
& =\{x \in U \mid \forall C \in \mathbf{C}(x \in C \Longrightarrow C \cap A \neq \emptyset)\} .
\end{aligned}
$$

For the maximum definition, we have:

$$
\begin{aligned}
\underline{a p r}^{M}(A) & =\operatorname{core}\left(\bar{\mu}_{A}\right) \\
& =\left\{x \in U \mid \bar{\mu}_{A}(x)=1\right\} \\
& =\{x \in U \mid \exists C \in \mathbf{C}(x \in C, C \subseteq A)\}, \\
& =\bigcup\{C \in \mathbf{C} \mid C \subseteq A\}, \\
\overline{\operatorname{apr}}^{M}(A) & =\operatorname{support}\left(\bar{\mu}_{A}\right) \\
& =\left\{x \in U \mid \bar{\mu}_{A}(x)>0\right\} \\
& =\{x \in U \mid \exists C \in \mathbf{C}(x \in C, C \cap A \neq \emptyset)\} \\
& =\bigcup\{C \in \mathbf{C} \mid C \cap A \neq \emptyset\} .
\end{aligned}
$$

The lower and upper approximations in each pair are no longer dual operators. However, $\left(\underline{a p r}{ }^{m}, \overline{a p r}^{M}\right)$ and $\left(\underline{a p r}^{M}, \overline{a p r}^{m}\right)$ are two pairs of dual operators. The first pair can be derived from the average definition, namely:

$$
\underline{a p r}^{*}(A)=\underline{a p r}^{m}(A), \quad \overline{a p r}^{*}(A)=\overline{a p r}^{M}(A) .
$$

These approximation operators have been studied extensively in rough set theory. Their connections and properties can be found in a recent paper by Yao [17]. 


\section{Conclusion}

By applying the techniques used in the study of rough sets, we propose a semantic framework for fuzzy sets. Two salient features of the proposed framework are the consideration of knowledge about the universe and the incorporation of a method for constructing fuzzy sets. This leads to a constructive fuzzy set theory, where fuzzy sets are explicitly constructed and interpreted.

Our examination of fuzzy sets and rough sets suggests that both theories share the same qualitative properties. The lower and upper approximations of rough set theory correspond to the core and support of fuzzy set theory. Several methods are suggested for the construction of fuzzy sets, depending on the available knowledge. Fuzzy membership functions are defined and interpreted in classical and generalized rough set models.

The connections established between the two theories may enhance our understanding of each theory. The results may provide a possible solution to a fundamental difficulty with fuzzy set theory regarding the semantic interpretations of fuzzy membership values.

\section{References}

1. Dubois, D. and Prade, P. A class of fuzzy measures based on triangular norms, International Journal of General Systems, 8, 43-61, 1982.

2. Dubois, D. and Prade, H. Rough fuzzy sets and fuzzy rough sets, International Journal of General Systems, 17, 191-209, 1990.

3. Dubois, D. and Prade, P. Similarity-based approximate reasoning, in: J.M. Zurada, R.J. Marks II, C.J. Robinson (Eds.), Computational Intelligence: Imitating Life, IEEE Press, New York, 69-80, 1994.

4. Dubois, D. and Prade, P. The three semantics of fuzzy set, Fuzzy Sets and Systems, 90, 141-150, 1997.

5. Klement, E.P., Mesiar, R. and Pap, E. Triangular Norms, Kluwer Academic Publishers, Dordrecht, 2000.

6. Klir, G.J. and Yuan, B. Fuzzy Sets and Fuzzy Logic: Theory and Applications, Prentice Hall, New Jersey, 1995.

7. Pawlak, Z. Rough sets, International Journal of Computer and Information Sciences, 11, 341-356, 1982.

8. Pawlak, Z. Rough Sets, Theoretical Aspects of Reasoning about Data, Kluwer Academic Publishers, Dordrecht, 1991.

9. Pawlak, Z. and Skowron, A. Rough membership functions, in: Advances in the Dempster-Shafer Theory of Evidence, R.R. Yager and M. Fedrizzi and J. Kacprzyk (Eds.), John Wiley and Sons, New York, 251-271, 1994.

10. Radzikowska, A.M. and Kerre, E.E. Fuzzy rough sets based on residuated lattices, in: Rough, Fuzzy and Fuzzy Rough Sets, D. Dubois, J. Grzymala-Busse, M. Inuiguchi, and L. Polkowski, Springer, Berlin, 2004.

11. Wong, S.K.M. and Ziarko, W. Comparison of the probabilistic approximate classification and the fuzzy set model, Fuzzy Sets and Systems, 21, 357-362, 1987.

12. Wu, W.Z. and Zhang, W.X. Neighborhood operator systems and approximations, Information Sciences, 144, 201-217, 2002. 
13. Yao, Y.Y. Two views of the theory of rough sets in finite universes, International Journal of Approximation Reasoning, 15, 291-317, 1996.

14. Yao, Y.Y. A comparative study of fuzzy sets and rough sets, Information Sciences, 109, 227-242, 1998.

15. Yao, Y.Y. Generalized rough set models, in: Rough Sets in Knowledge Discovery, Polkowski, L. and Skowron, A. (Eds.), Physica-Verlag, Heidelberg, 286-318, 1998.

16. Yao, Y.Y. Constructive and algebraic methods of the theory of rough sets, Information Sciences, 109, 21-47, 1998.

17. Yao, Y.Y. Relational interpretations of neighborhood operators and rough set approximation operators, Information Sciences, 111, 239-259, 1998.

18. Yao, Y.Y. Rough sets and interval fuzzy sets, Proceedings of Joint 9th IFSA World Congress and 20th NAFIPS International Conference, 2347-2352, 2001.

19. Yao, Y.Y. Information granulation and approximation in a decision-theoretic model of rough sets, manuscript, 2002.

20. Yao, Y.Y. and Lin, T.Y. Generalization of rough sets using modal logic, Intelligent Automation and Soft Computing, an International Journal, 2, 103-120, 1996.

21. Yao, Y.Y. and Wong, S.K.M. A decision theoretic framework for approximating concepts, International Journal of Man-machine Studies, 37, 793-809, 1992.

22. Yao, Y.Y. and Zhang, J.P. Interpreting fuzzy membership functions in the theory of rough sets, Proceedings of The Second International Conference on Rough Sets and Current Trends in Computing, LNAI 2005, 82-89, 2001.

23. Zadeh, L.A. Fuzzy sets, Information and Control, 8, 338-353, 1965.

24. Zakowski, W. Approximations in the space $(U, \Pi)$, Demonstratio Mathematica, XVI, 761-769, 1983.

25. Zhang, B. and Zhang, L. Theory and Applications of Problem Solving, NorthHolland, Amsterdam, 1992. 